\title{
Self-assembling tensor networks and holography in disordered spin chains
}

\author{
Andrew M. Goldsborough* and Rudolf A. Römer ${ }^{\dagger}$ \\ Department of Physics and Centre for Scientific Computing, The University of Warwick, Coventry CV4 7AL, United Kingdom
}

(Received 17 January 2014; published 27 June 2014)

\begin{abstract}
We show that the numerical strong disorder renormalization group algorithm of Hikihara et al. [Phys. Rev. B 60, 12116 (1999)] for the one-dimensional disordered Heisenberg model naturally describes a tree tensor network (TTN) with an irregular structure defined by the strength of the couplings. Employing the holographic interpretation of the TTN in Hilbert space, we compute expectation values, correlation functions, and the entanglement entropy using the geometrical properties of the TTN. We find that the disorder-averaged spin-spin correlation scales with the average path length through the tensor network while the entanglement entropy scales with the minimal surface connecting two regions. Furthermore, the entanglement entropy increases with both disorder and system size, resulting in an area-law violation. Our results demonstrate the usefulness of a self-assembling TTN approach to disordered systems and quantitatively validate the connection between holography and quantum many-body systems.
\end{abstract}

DOI: $10.1103 /$ PhysRevB.89.214203

PACS number(s): 75.10.Jm, 05.30.-d, 02.70.-c

\section{INTRODUCTION}

There is currently a lot of excitement around the so-called Anti-de Sitter/conformal field theory (AdS/CFT) correspondence and possible applications in condensed matter physics [1]. The AdS/CFT correspondence is most well known in high energy physics where it was noted [2] that there exists a duality between certain theories of gravity on $(D+1)$ dimensional AdS space-time and conformal quantum field theories (CFT) living on its $D$-dimensional boundary. In condensed matter systems, the AdS/CFT correspondence can provide a geometric interpretation of renormalization group (RG) techniques since the additional holographic dimension can be interpreted as a scale factor in the RG coarse graining [1]. It has been argued recently $[3,4]$ that certain RG approaches to the Hilbert space of critical many-body interacting system in $D$ dimensions, such as the multiscale entanglement renormalisation ansatz for tensor networks, share many of their geometric properties with $(D+1)$-dimensional AdS. This connection is based on ideas [5] that suggest that the entanglement entropy of a region on the boundary is related to the minimal surface in the holographic bulk that separates the region from the rest of the surface. These ideas were further developed by Evenbly and Vidal [6] to discuss the underlying geometric structure of entanglement and correlation functions in such tensor networks in general.

Tensor network methods provide elegant and powerful tools for the simulation of quantum many-body systems. Their original manifestation, the density-matrix renormalization group (DMRG) [7], is now understood to be based on a variational update of a matrix product state (vMPS) [8,9], and has found

\footnotetext{
*a.goldsborough@warwick.ac.uk; www.warwick.ac.uk/andrewgoldsborough.

†r.roemer@warwick.ac.uk; www.warwick.ac.uk/rudoroemer.
}

Published by the American Physical Society under the terms of the Creative Commons Attribution 3.0 License. Further distribution of this work must maintain attribution to the author(s) and the published article's title, journal citation, and DOI. applications in a wide range of fields such as quantum chemistry [10] and quantum information [11] as well as condensed matter physics [12]. More recent developments have extended the methods to, e.g., critical systems [13], two-dimensional lattices [14-16], and topologically ordered states [17].

For disordered quantum many-body systems, the strongdisorder renormalization group (SDRG) provides a similarly unifying approach [18]. It was originally devised by Ma, Dasgupta, and $\mathrm{Hu}[19,20]$ for the random antiferromagnetic (AFM) Heisenberg chain

$$
H=\sum_{i=1}^{L-1} J_{i} \vec{s}_{i} \cdot \vec{s}_{i+1},
$$

where $\vec{s}_{i}$ is the spin-1/2 operator and $J_{i}$ is the coupling constant, which takes a random value between $0<J_{i}<J_{\max }$ according to some probability distribution $P(J)$. The principle behind the SDRG is to eliminate the most strongly coupled pairs of spins and replace them with an effective interaction that couples the spins at either side of the pair, as shown in Fig. 1(a). The pair of spins coupled by $J_{\max }$ are thought as being frozen into a singlet ground state as the neighboring interactions are significantly weaker - ultimately leading to the random singlet phase, which is the ground state of the system [21,22]. This freezing of degrees of freedom is remarkably close to an update process in entanglement RG for tensor networks [13] and suggests the possible usefulness of the AdS/CFT correspondence also for disordered spin chains. By analyzing the probability of survival through the SDRG algorithm it is possible to predict that mean correlations will have a power-law decay [21] with negative power 2. Similarly, the entanglement entropy can be shown to scale logarithmically with block size [23], where the amount of entanglement between blocks $A$ and $B$ is quantified by the von Neumann entropy

$$
S_{A \mid B}=-\operatorname{Tr} \rho_{A} \log _{2} \rho_{A},
$$

with $\rho_{A}$ the reduced density matrix obtained by tracing over the $B$ components of the density matrix.

In this work, we have developed a self-assembling tree tensor network (TTN) algorithm based on the previous ideas of SDRG [24,25]. This allows us to calculate properties such 


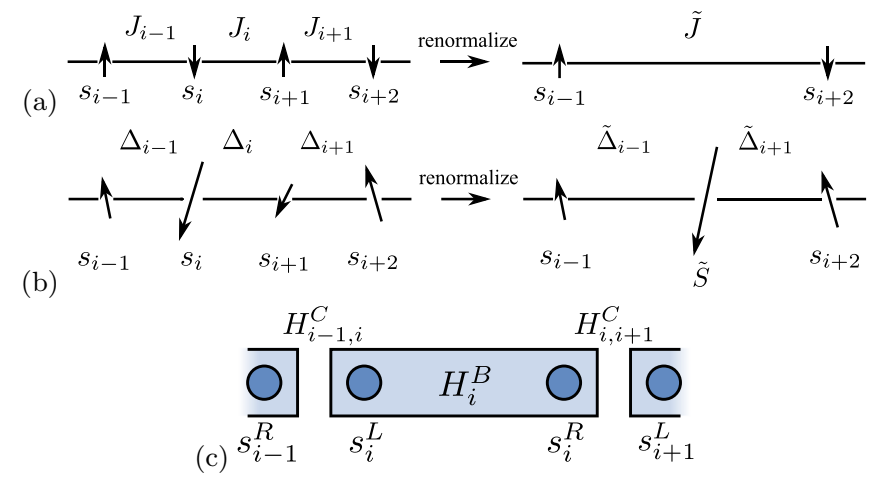

FIG. 1. (Color online) Schematic diagrams of the various SDRG variants. Horizontal lines indicate the one-dimensional spin system. (a) Traditional MDH SDRG [19], spins $\vec{s}_{i}, \vec{s}_{i+1}$ (arrows) with the greatest coupling strength, $J_{i}>J_{k} \forall k \neq i$, are removed and replaced by an effective coupling $\tilde{J}$. (b) SDRG of Westerberg et al. [24]; spin pairs are renormalized for the largest energy gap $\Delta_{i}$ and replaced by an effective spin $\tilde{S}$. (c) SDRG variant of Hikihara et al. [25]; the chain is decomposed into blocks of spins described by block Hamiltonians $H^{B}$ (shaded rectangles), with left and right spins, respectively, $s^{L}$ and $s^{R}$ (dark dots) on the boundaries of the blocks forming the coupling Hamiltonians, $H^{C}$.

as expectation values, correlation functions, and entanglement entropy directly and efficiently from the geometry of the TTN. In particular, we find that the distance dependence of the spin-spin correlation function can be studied not only via direct calculation of the correlation functions, but also via the holographic distance dependence along the tree network connecting two sites. In Sec. II we will briefly review the numerical strong disorder renormalization group of Hikihara et al. [25] and define the states and operators that form the basis for our work. Section III shows how the numerical SDRG on a matrix product operator (MPO) self-assembles the TTN. Finally, in Sec. IV we compute correlation functions and entanglement entropy (i) directly using the TTN as well as (ii) via simply counting the path lengths and connectivities in the holography. We find that both approaches give consistent results.

\section{THE MPO IMPLEMENTATION OF THE SDRG}

\section{A. The numerical SDRG}

The SDRG method was extended to both ferromagnetic (FM) and AFM couplings by Westerberg et al. [24,26]. The approach finds the neighboring pair of spins $\vec{s}_{i}, \vec{s}_{i+1}$ with the greatest energy gap $\Delta_{i}$ between the ground state and excited state and combines them into a single effective spin $\tilde{S}$ [Fig. 1(b)]. The effective couplings between the new spin and its neighbors are then recalculated using Clebsch-Gordan coefficients and the new gaps $\tilde{\Delta}_{i-1}$ and $\tilde{\Delta}_{i}$ updated. SDRG was once more extended by Hikihara et al. [25] to include higher states at each decimation, in the spirit of the numerical renormalization group [27] and the DMRG [7]. This method therefore decomposes the system into blocks rather than larger spins allowing for more accurate computation of, e.g., the spin-spin correlation functions. The more states that are kept at each decimation the more accurate the description and is exact in the limit of all states kept.

Consider a point in the algorithm where the Hamiltonian is made up of blocks $H_{i}^{B}$ at each site and couplings $H_{i, i+1}^{C}$ between them as in Fig. 1. The couplings take the form of a two spin Hamiltonian

$$
H_{i, i+1}^{C}=J_{i} \vec{s}_{i}^{R} \cdot \vec{s}_{i+1}^{L},
$$

where $\vec{s}_{i}^{R}$ is the spin operator of the right-hand spin of block $i$ and $\vec{s}_{i+1}^{L}$ is the left-hand spin of block $i+1$. In full the Hamiltonian is

$$
H=\sum_{i=1}^{N_{B}} H_{i}^{B}+\sum_{i=1}^{N_{B}-1} H_{i, i+1}^{C},
$$

where $N_{B}$ is the number of blocks.

Let us now define the gap $\Delta_{i}$ as the energy difference between the highest energy $\mathrm{SU}(2)$ multiplet that would be kept and the smallest multiplet that would be discarded in a renormalization of block $H_{i, i+1}^{B}$. The scheme works by searching for the pair of blocks with the largest gap $\Delta_{i_{m}}$ and then combines the coupling and the blocks that it connects into a single block

$$
H_{i_{m}, i_{m}+1}^{B}=H_{i_{m}}^{B}+H_{i_{m}, i_{m}+1}^{C}+H_{i_{m}+1}^{B} .
$$

This block and the couplings either side are then renormalized by a matrix $\left(V_{\chi}\right)$ of the eigenvectors corresponding to the lowest $\chi$ eigenvalues of the block, such that only full SU(2) blocks are kept. The process is repeated until the system is represented by one block. The details of the algorithm are described in Appendix A.

\section{B. Numerical SDRG as an MPO process}

Hikihara's numerical SDRG can be naturally described as a set of operations on an MPO (see Appendix B for more details). First, we contract the MPO tensors for the pair of sites with the largest gap, sites $i_{m}$ and $i_{m}+1$ [Fig. 2(a)],

$$
W^{\left[i_{m}, i_{m}+1\right]}=\sum_{b_{i_{m}}} W_{b_{i_{m}-1}, b_{i_{m}}}^{\sigma_{i_{m}}, \sigma_{i^{\prime}}^{\prime}} W_{b_{i_{m}}, b_{i_{m}+1}}^{\sigma_{i_{m+1}}, \sigma_{i_{m}+1}^{\prime}} .
$$

Here we have $\sigma_{i_{m}}=1, \ldots, \chi$ for the physical indices, and for the Heisenberg model (1), the virtual indices are $b_{i_{m}}=$ $1, \ldots, 5$. Next, we perform an eigenvalue decomposition on the on-site components of the new MPO tensor keeping the eigenvectors of the lowest $\chi$ eigenvalues $\left(V_{\chi}\right)$

$$
\Lambda_{\chi}=V_{\chi}^{\dagger}\left(H_{i_{m}}^{B} \otimes \mathbb{1}+J_{i_{m}} \vec{s}_{i_{m}}^{R} \cdot \vec{s}_{i_{m}+1}^{L}+\mathbb{1} \otimes H_{i_{m}+1}^{B}\right) V_{\chi} .
$$

As with the Hikihara's algorithm, only the $\chi$ eigenvalues that make up full SU(2) multiplets are used. Then we contract $V_{\chi}$ and $V_{\chi}^{\dagger}$ with the new MPO tensor to perform the renormalization [Fig. 2(b)]. For the moment write the two-site combined MPO $W^{\left[i_{m}, i_{m}+1\right]}$ in terms of an effective site with index $\tau=1, \ldots, \chi, \chi+1, \ldots, \chi^{2}$, i.e., $W_{b_{i_{m}-1}, b_{i_{m}+1}}^{\tau, \tau^{\prime}}$. Similarly, we can write the set of eigenvectors as $\left[V_{\chi}\right]_{\tau}^{\tilde{\sigma}_{i m}}$. Then the contraction is explicitly given as

$$
W_{b_{i_{m}-1}, b_{i m}}^{\tilde{\sigma}_{i_{m}}, \tilde{\sigma}_{i_{m}}^{\prime}}=\sum_{\tau, \tau^{\prime}}\left[V_{\chi}^{\dagger}\right]_{\tau}^{\tilde{\sigma}_{i_{m}}} W_{b_{i_{m}-1}, b_{i_{m}}}^{\tau, \tau^{\prime}}\left[V_{\chi}\right]_{\tau^{\prime}}^{\tilde{\sigma}_{i m}^{\prime}}
$$


(a)

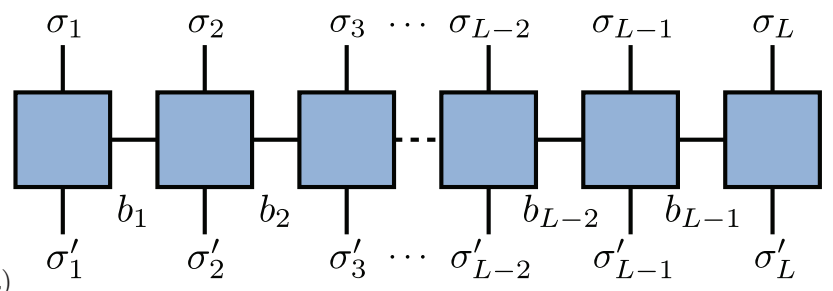

(b)

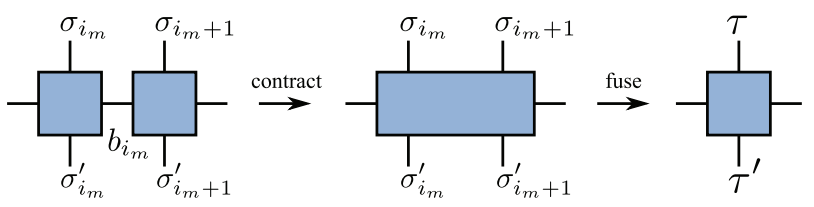

(c)

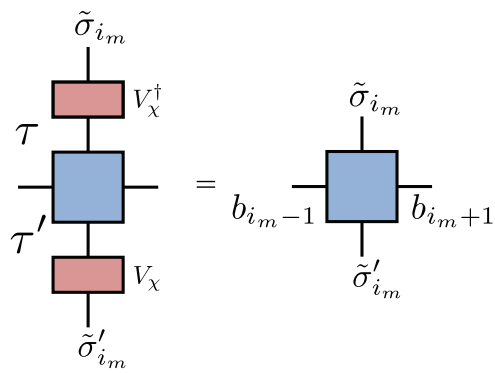

FIG. 2. (Color online) (a) Tensor network diagram of the matrix product operator. The (vertical) $\sigma$ and $\sigma^{\prime}$ legs denote physical indices and couple to the tensor network wave function and conjugate. The $b$ 's are virtual indices (in horizontal direction) and couple the local tensors (blue-shaded squares) of the MPO to each other. (b) The pair of sites with the largest gap $\Delta_{i_{m}}$ is found, the MPO tensors for these sites are contracted, and the physical indices fused to form a matrix. (c) Contracting the matrices of eigenvectors $V_{\chi}$ (red-shaded rectangle) and $V_{\chi}^{\dagger}$ creates a new MPO for a coarse-grained system.

where $\tilde{\sigma}_{i_{m}}=1, \ldots, \chi$ is the spin index of the renormalized site $i_{m}$. Hence we replace sites $i_{m}$ and $i_{m}+1$ with a single renormalized site and relabel the remaining indices.

The contraction makes the on-site component of the new MPO simply a diagonal matrix of the lowest $\chi$ eigenvalues $\Lambda_{\chi}$ [Fig. 3(a)]. It also has the effect of renormalizing the coupling spins just as in the Hikihara approach [Fig. 3(b)]:

$$
\begin{gathered}
\overrightarrow{\tilde{s}}_{i_{m}}^{R}=V_{\chi}^{\dagger}\left(\mathbb{1} \otimes \vec{s}_{i_{m}+1}^{R}\right) V_{\chi}, \\
\overrightarrow{\tilde{s}}_{i_{m}}^{L}=V_{\chi}^{\dagger}\left(\vec{s}_{i_{m}}^{L} \otimes \mathbb{1}\right) V_{\chi} .
\end{gathered}
$$

The contraction therefore maps two MPO tensors onto one while preserving the indexing structure of the MPO.

As the final step, we diagonalize the neighboring blocks to update the distribution of gaps. The procedure is then repeated until the system is just one site, and we diagonalize to obtain the ground-state energy $E_{g}$ of the system.

\section{TREE TENSOR NETWORKS AND SDRG}

The MPO description of SDRG given above amounts to a coarse-graining mechanism that acts on the operator. Alternatively, we can view it as a multilevel tensor network wave function acting on the original operator. To illustrate this, we can split the $\tau$ index of $V_{\chi}^{\dagger}$ as in Eq. (8) back to the original spin indices $\sigma_{i_{m}}, \sigma_{i_{m}+1}$ to create an isometric tensor or isometry (a)
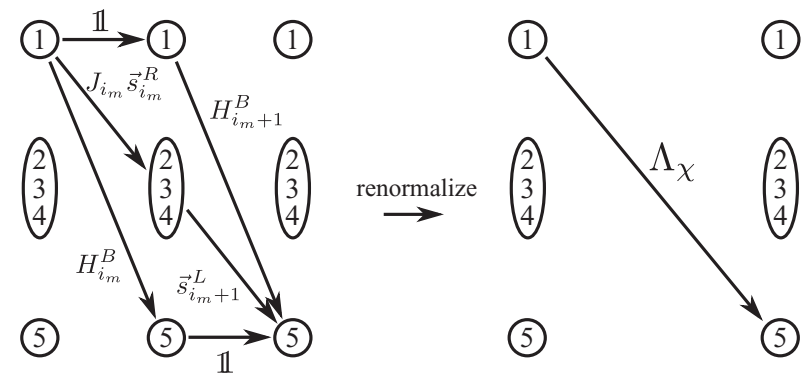

(b)
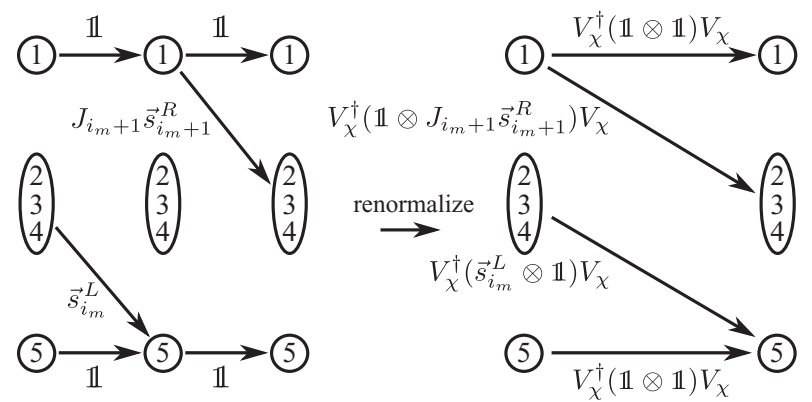

FIG. 3. Schematic representation of the contraction step (8) for the Heisenberg Hamiltonian (1). Circles (and ellipses) denote (combined) operator entries in the Heisenberg MPO $W^{[i, i+1]}$ (see Appendix B 2 for details). (a) Renormalizing the on-site components has the effect of creating a new on-site component, which is a diagonal matrix of the lowest eigenvalues $\Lambda_{\chi}$. (b) Contracting $V_{\chi}$ and $V_{\chi}^{\dagger}$ has the effect of renormalizing the coupling spins in the same way as the Hikihara method, storing them as the coupling components of the new MPO tensor.

$\left[V_{\chi}^{\dagger}\right]_{\tau}^{\tilde{\sigma}_{i m}} \equiv[w]_{\sigma_{i_{m}}, \sigma_{i_{m}+1}}^{\tilde{\sigma}_{i}}[28]$. The isometric property means that

$$
\sum_{\sigma_{i_{m}}, \sigma_{i_{m}+1}}[w]_{\sigma_{i_{m}}, \sigma_{i_{m}+1}}^{\tilde{\sigma}_{i_{m}}}\left[w^{\dagger}\right]_{\sigma_{i_{m}}, \sigma_{i_{m}+1}}^{\tilde{\sigma}_{i^{\prime}}^{\prime}}=\delta^{\tilde{\sigma}_{i_{m}}, \tilde{\sigma}_{i_{m}}^{\prime}}
$$

or $w w^{\dagger}=\mathbb{1} \neq w^{\dagger} w$ [Fig. 4(a)]. A renormalization in the SDRG algorithm as in Figs. 2(b) and 2(c) can then be rephrased graphically as in Fig. 4(b). This makes the notion of mapping two MPO tensors to one immediately explicit.

When viewed in terms of isometries, the algorithm can be seen to self-assemble a tensor network based on the positions of largest gaps before each renormalization. When written in full, it builds an inhomogeneous binary TTN as shown in Fig. 5.

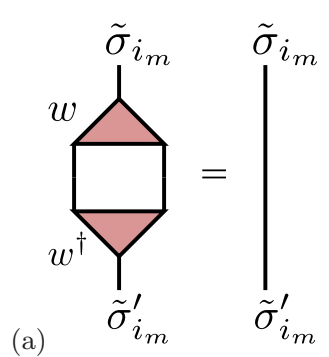

(b)

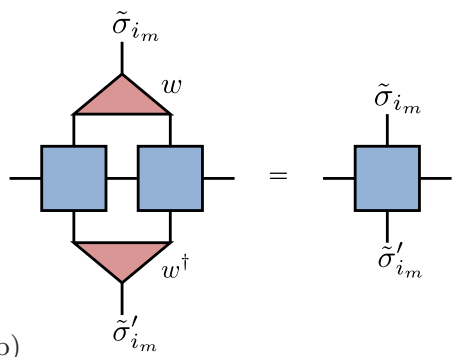

FIG. 4. (Color online) (a) Schematic representation of the isometric property $w w^{\dagger}=\mathbb{1}$ given by Eq. (11). (b) One step in the MPO SDRG algorithm in terms of isometric tensors $w$. Triangles (red-shaded) denote the isometries; squares are as in Fig. 2. 


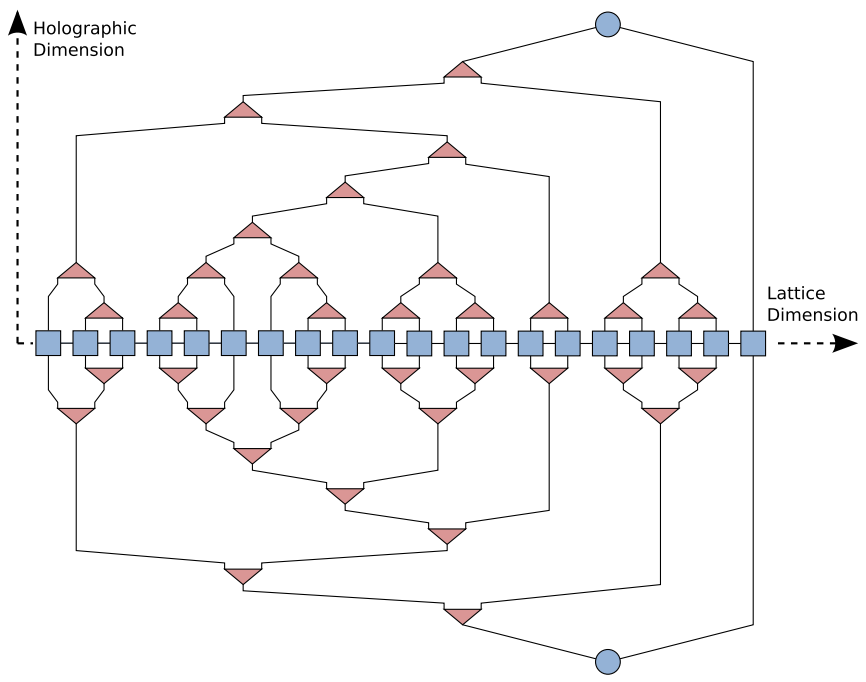

FIG. 5. (Color online) The SDRG algorithm as a TTN for a chain of $L=20$ sites. The squares are the MPOs (i.e., the spin operators), triangles are isometric tensors, and solid lines denote summations over physical (vertical) and virtual (horizontal) indices as before. The circle indicates the top tensor, i.e., the ground-state eigenvector of the coarse-grained system. Lattice and holographic dimensions are indicated by the dashed arrows.

Tree tensor networks are one of the major areas of tensor network research and TTNs with regular structures have been extensively studied [29-32]. The isometric nature of the isometries allows for calculations to be performed in a highly efficient manner [28,30,33]. When calculating expectation values, such as the two-point correlation function [Fig. 6(a)], only those tensors that affect the sites that the operators act on need to be included; this is known as the past causal cone [28] and is drawn as a blue shadow in the holographic bulk. This allows for a reduction in the number of contractions that need to be performed to obtain a result. Calculation of the entanglement (von Neumann) entropy (2) can also be made more efficient as shown in Fig. 6(b). In addition to the reduction due to the isometries, we note that the entanglement entropy is not affected by the isometries acting just on $A$ [30]. However, the entries in the density matrix will change so we label it $A^{\prime}$.

\section{RESULTS}

In the following, we shall compare results for the disordered antiferromagnetic Heisenberg model (1) when using a modern DMRG implementation, e.g., variational MPS (vMPS), with those obtained from our TTN SDRG strategy (tSDRG). The set of couplings $\left(J_{i}\right)$ shall always be taken from a box-type distribution [25], i.e., constant in the range $0<1-\Delta J / 2<$ $J_{i}<1+\Delta J / 2<2$ and zero outside. Unless stated otherwise, we use strong disorder $\Delta J=2^{-}$in the following. We assume open (hard wall) boundary conditions throughout.

\section{A. Convergence and ground-state energies}

In Fig. 7 (main), we show the dependence of the disorderaveraged ground-state energy per site, $E_{g} / L$, on $\Delta J$ for constant $L$. We find that for both vMPS and tSDRG, the $E_{g} / L$ values decrease for increasing $\Delta J$, i.e., the ground-state energy lowers as disorder in the $J_{i}$ couplings allows the system to form particularly energetically favorable spin configurations. We also see that the vMPS for the chosen values of $\chi$ and $L$ reaches lower energies. This suggests that it is yet more efficient in finding an approximation to the true ground-state energy. However, upon increasing $\Delta J$, the difference between vMPS and tSDRG is getting smaller. This is expected since SDRG is based on the idea that the contribution from the nonsinglet interactions is small, which is more accurate an assumption the greater the disorder. The figure also shows that increasing $\chi$ can considerably improve the results of the tSDRG [34].

In Fig. 7 (inset) we show $E_{g} / L$ as a function of $L$ for various values of $\chi$ at the strongest permissible disorder $\Delta J=2^{-}$. We find that the values of $E_{g} / L$ do not vary much anymore for system sizes $L \geqslant 100$. Conversely, $E_{g} / L$ values for $L<100$ are clearly dominated by the presence of the open boundary conditions.

\section{B. Correlation functions}

The correlation functions for a strongly disordered Heisenberg chain are expected to average out to be a power-law decay [21]

$$
\left\langle\left\langle\vec{s}_{x_{1}} \cdot \vec{s}_{x_{2}}\right\rangle\right\rangle \sim \frac{(-1)^{x_{2}-x_{1}}}{\left|x_{2}-x_{1}\right|^{2}},
$$
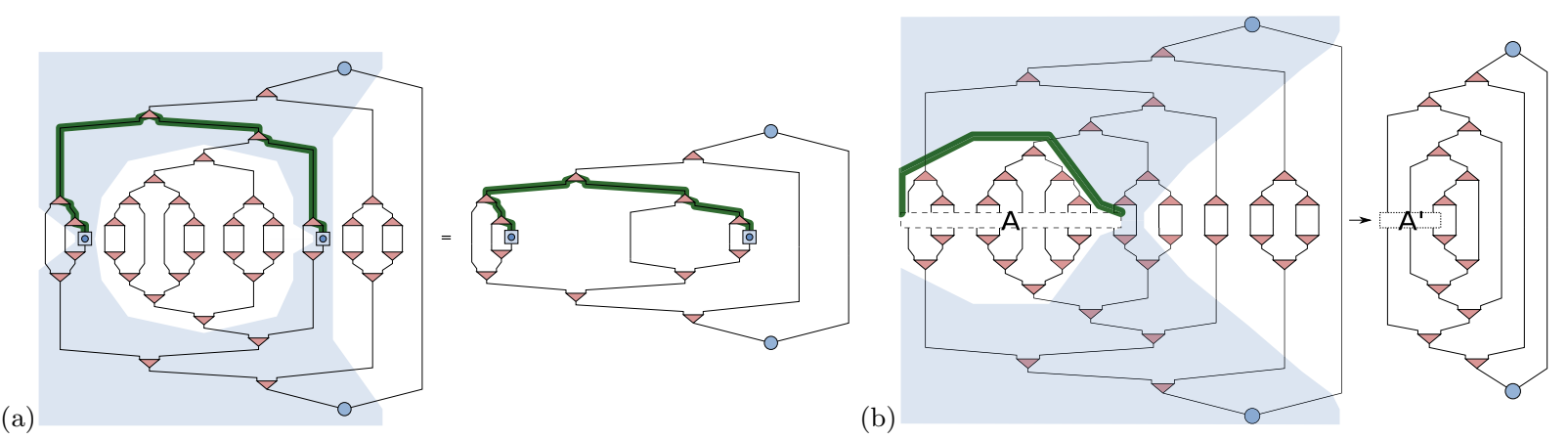

FIG. 6. (Color online) Diagram showing the TTN form of (a) the correlation function $\left\langle\vec{s}_{3} \cdot \vec{s}_{15}\right\rangle$ and (b) the reduced density matrix $\rho_{A}$ (for the block $A$ indicated by the dashed rectangle ten sites long) of the 20 site system from Fig. 5. Lines and symbols as in Fig. 5. The causal cone in both panels is indicated by a light-blue-shaded region. The bold line in panel (a) shows the path length through the TTN connecting the two sites, whereas the bold line in (b) shows the minimal surface in the TTN between regions $A$ and $B$ (the rest of the chain). The diagram in the right-hand side of (b) has been reduced in the horizontal direction to highlight the reduction in complexity due to the isometries. 


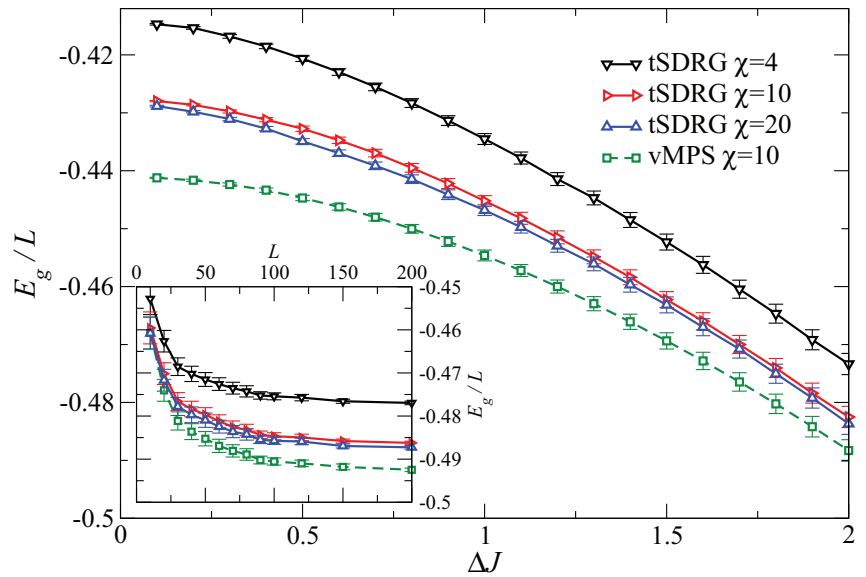

FIG. 7. (Color online) Ground-state energy per site $E_{g} / L$ as a function of disorder $\Delta J$ for system size $L=100$ for tSDRG (solid lines) and variational MPS (dashed). The error bars correspond to the standard error on the mean obtained from averaging over 200 different disorder configurations and various values of $\chi$. Lines are guides to the eye. Inset: System size dependence of $E_{g} / L$ for $\Delta J=2^{-}$. Sizes $L=10-80$ have been averaged over 500 disorder configurations, 90, 100, and 120 over 1000, 150 and 200 over 2000 configurations, respectively.

where $\left\langle\left\langle\vec{s}_{x_{1}} \cdot \vec{s}_{x_{2}}\right\rangle\right\rangle$ is understood to be the disorder-averaged expectation value of the two-point spin-spin correlation function. This $r^{-2}$ scaling of the correlation is a feature of the disorder in the system [21] and should be contrasted with the well-known power-law dependence of correlation functions [35] in interaction-driven Luttinger liquids [36].

In loop-free tensor networks, correlations scale as $e^{-\alpha D\left(x_{1}, x_{2}\right)}$, where $D\left(x_{1}, x_{2}\right)$ is the number of tensors that connect site $x_{1}$ to $x_{2}$ [6]. DMRG is based on the matrix product state (MPS) and as such it has one tensor per site, i.e., $D_{\text {MPS }} \approx\left|x_{2}-x_{1}\right|$. Therefore correlations in DMRG scale exponentially. This suggests that for long chains it will be necessary to keep large numbers of states to be able to model a power-law correlation of the system [6]. tSDRG, on the other hand, has a holographic geometry based on a random TTN with path length $D_{\mathrm{TTN}} \approx \log \left|x_{2}-x_{1}\right|$, i.e., scaling logarithmically with distance when averaged. This makes it much more suited to capture the desired power-law decay

$$
\begin{aligned}
\left\langle\left\langle\vec{s}_{x_{1}} \cdot \vec{s}_{x_{2}}\right\rangle\right\rangle & \sim e^{-\alpha\left\langle D_{\operatorname{TTN}}\left(x_{1}, x_{2}\right)\right\rangle} \\
& \sim e^{-\alpha \log \left|x_{2}-x_{1}\right|} \sim\left|x_{2}-x_{1}\right|^{-a} .
\end{aligned}
$$

In Fig. 8, we show the behavior of $\left\langle\left\langle\vec{s}_{x_{1}} \cdot \vec{s}_{x_{2}}\right\rangle\right\rangle$ computed directly as well as its holographic estimate based on (13). We find that the behavior for $\left|x_{2}-x_{1}\right| \gg 1$ and $\left|x_{2}-x_{1}\right|<$ $L / 2$ is indeed very similar for both approaches. The best-fit value for $\alpha$ is $0.62 \pm 0.02$ where the error is the standard error [37]. We find that in the indicated distance regime, both measures of $\left\langle\left\langle\vec{s}_{x_{1}} \cdot \vec{s}_{x_{2}}\right\rangle\right\rangle$ are consistent with the expected $r^{-2}$ behavior. For $\left|x_{2}-x_{1}\right| \gtrsim L / 2$ we see that the boundaries lead to an upturn on the behavior of $\left\langle\left\langle\vec{s}_{x_{1}} \cdot \vec{s}_{x_{2}}\right\rangle\right)$ for both direct and holographic estimates. This upturn is a result of boundary effects and can easily be understood in terms of the holographic TNN: For $\left|x_{2}-x_{1}\right| \geqslant L / 2$, the average path length in the tree

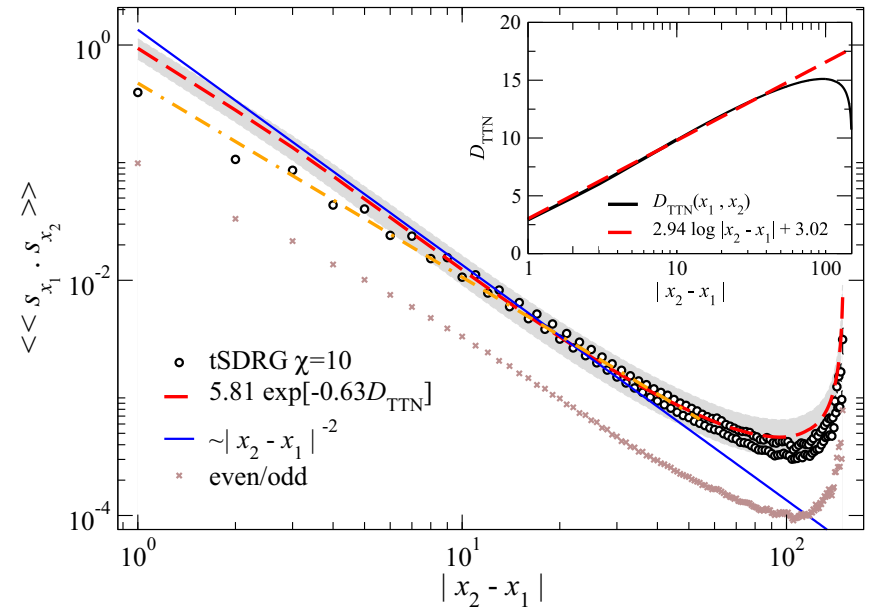

FIG. 8. (Color online) Correlation function for $L=150$ and $\Delta J=2^{-}$averaged over 2000 samples for the direct calculation of $\left\langle\left\langle\vec{s}_{x_{1}} \cdot \vec{s}_{x_{2}}\right\rangle\right\rangle$ (black circles) and also via the holographic approach (13) using $D_{\text {TTN }}$ (dashed red line with error of mean indicated by the gray shading) such that $\left\langle\left\langle\vec{s}_{x_{1}} \cdot \vec{s}_{x_{2}}\right\rangle\right\rangle(5.81 \pm 0.93) \exp [-(0.62 \pm$ $\left.0.02) D_{\text {TTN }}\right]$. The expected thermodynamic scaling $\left|x_{2}-x_{1}\right|^{-2}$ is also shown (solid blue line) while the dashed orange line denotes a power-law fit up to $\left|x_{2}-x_{1}\right|=50$ with slope 1.64 . The (brown) crosses show $\left\langle\left\langle\vec{s}_{x_{1}} \cdot \vec{s}_{x_{2}}\right\rangle\right\rangle / 4$ (for clarity) with all values for even distances $\left|x_{2}-x_{1}\right|$ multiplied by 1.25 . Inset: The holographic path length $D_{\text {TTN }}$ connecting sites $x_{1}$ and $x_{2}$ averaged over the 2000 TTNs (black) and a fit in the logarithmic regime (red).

decreases (cf. Fig. 6). This is also consistent with periodic systems where we expect correlation functions to be equal for $\left|x_{2}-x_{1}\right|=r$ and $L-r$. In the inset of Fig. 8 we show the distance dependence of $D_{\mathrm{TTN}}$ with $\chi=10$. For $\left|x_{2}-x_{1}\right|<$ $L / 2$, the data can be described as linear behavior in $\log \mid x_{2}-$ $x_{1} \mid$ with slope $2.94 \pm 0.02$. Note that this slope along with the value of $\alpha=0.62 \pm 0.01$ gives an estimate of power-law exponent $a=0.62 \times 2.94=1.84 \pm 0.04$ for fixed $L=150$. Figure 9 shows that as $L$ increases, the resulting value of the

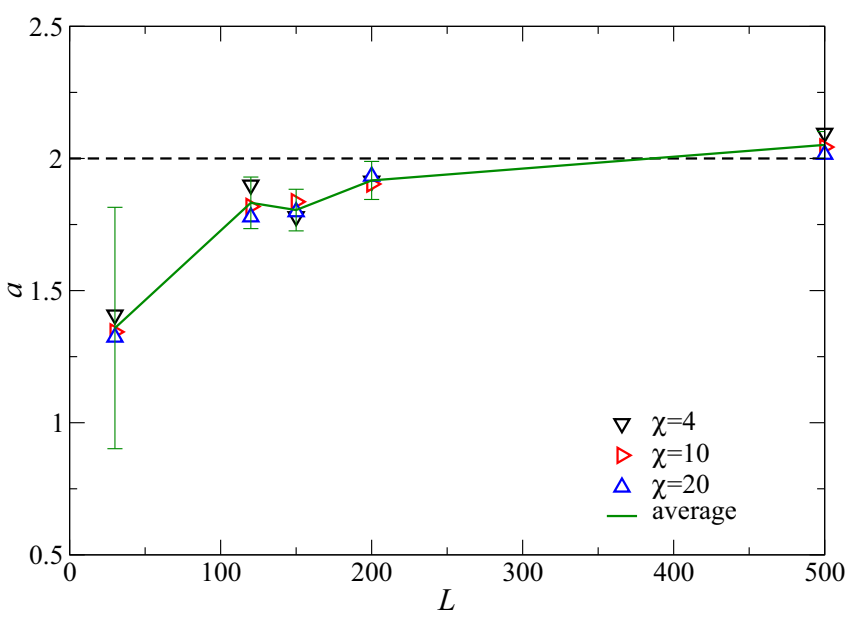

FIG. 9. (Color online) The scaling parameter $a$ from Eq. (13) as a function of system size $L$ for different values of $\chi$ at $\Delta J=2^{-}$. The solid lines are guides to the eye only. The asymptotic value of $a=2$ is indicated by the horizontal dashed line. 


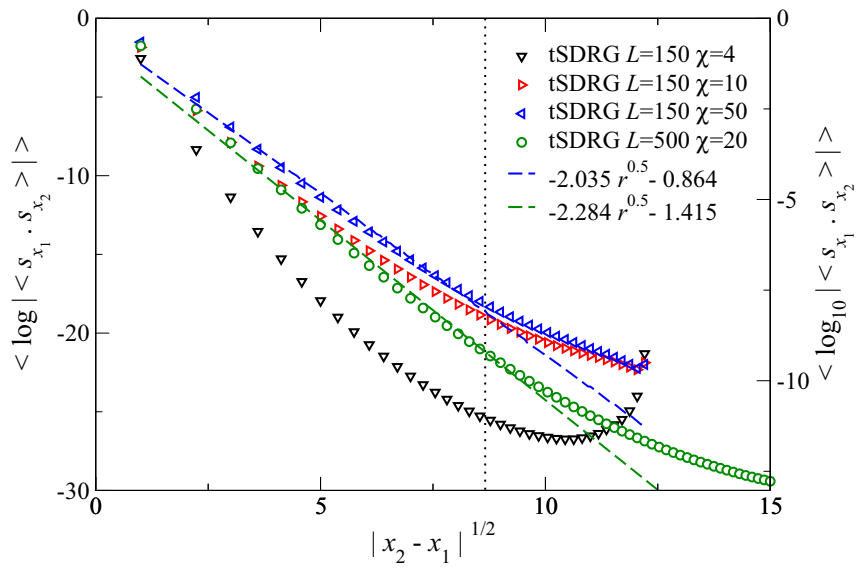

FIG. 10. (Color online) The typical spin correlation function averaged over 2000 samples for $L=500$ (green circles) and $L=150$ (triangles) and $\chi$ values as given in the legend. Error bars are within symbol size throughout. The dashed lines are fits to the linear regimes for $L=150, \chi=50$ (blue) and $L=500, \chi=20$ (green). The vertical dotted line indicates half the system size for $L=150$.

scaling power $a$ also increases towards the expected value of 2 for larger systems upon increasing $L$. We have also checked that the differences between $\chi=10$ and 20 remain within the error bars and hence we use $\chi=10$ for calculations of $\left\langle\left\langle\vec{s}_{x_{1}} \cdot \vec{s}_{x_{2}}\right\rangle\right\rangle$ in Fig. 8. We further note that Fig. 8 shows a clear difference in the correlation function between even and odd distances. The difference in magnitude is found to be $1 / 4$ as predicted previously [38].

In addition to the power-law scaling of mean correlations, it is expected [21] that the typical correlations scale as

$$
\left\langle\log \left|\left\langle\vec{s}_{x_{1}} \cdot \vec{s}_{x_{2}}\right\rangle\right|\right\rangle \sim-\left|x_{2}-x_{1}\right|^{1 / 2},
$$

where the left-hand side of (14) is the disorder-averaged mean of the logarithm of the spin correlation function, i.e., the logarithm of the geometric mean of the correlation function. Figure 10 shows that this typical correlation function indeed scales as $\left|x_{2}-x_{1}\right|^{1 / 2}$ and the quality of the fit increases upon increasing $\chi$ and system size. For $L=150$, as $\chi$ is increased from 4 to 50, the agreement with (14) improves up to approximately half the system size, at which point boundary effects become important as in Fig. 8. The typical/geometric mean of the path lengths does not allow one to reproduce the typical correlation behavior (14), but rather continues to retain a logarithmic scaling behavior. This suggests that the TTN constructed by our tSDRG selects those path lengths corresponding to mean correlation. Clearly, Eq. (13) ignores correlation information stored in the isometry tensors and we expect that its inclusion will recover also the typical correlation behavior. Indeed, the need to increase $\chi$ in Fig. 10 in order to reproduce (14) already confirms that the tensor content is very important here.

\section{Entanglement entropy}

In general, the entanglement entropy $S_{A \mid B}$ is difficult to compute as the size of the reduced density matrix $\rho_{A}$ scales exponentially with the size of block $A$. While for special cases, such as the $X X$ model [39], $S_{A \mid B}$ can be computed more easily, the general strategy involves finding the eigen- or singular values of $\rho_{A}$ [9].

The TTN representation of tSDRG gives an alternative means of calculating $S_{A \mid B}$ for any bipartitions $A$ and $B$ of the system. In a similar manner to the correlation functions, the geometry of the tensor network is related to its ability to capture $S_{A \mid B}$. Briefly, $S_{A \mid B}$ is proportional to the minimum number of indices, $n_{A}$, that one would have to cut to separate a block $A$ of spins from the rest $B$ of the chain (cf. Fig. 6) [6]. This dependence is related to the famous area law, which states that for the ground state of a gapped system, the entanglement entropy of a region is proportional to the size of the boundary that separates the two regions [40,41]. The MPS is a simple line of tensors (cf. Appendix B) and thus the number of indices that separate one region from another is a constant and independent of the size of the block and its position in the chain. Unlike the MPS, for the TTN the position of the block in the chain alters the number of indices that have to be cut to separate it from the rest of the system. This suggests that there are spatial regions in the chain that are more and less entangled, which is likely to be true for a strongly disordered spin chain. The concept is hence similar to discussing the entanglement in the Ma, Dasgupta, and Hu implementation [19] of SDRG, where the entanglement entropy is related to the number of singlets that have to be broken to separate a region from the rest [23].

In Fig. 11 we show that the average value of $S_{A \mid B}$ remains approximately constant upon increasing the disorder, while the average of the maximal $S_{A \mid B}$ shows a pronounced increase. This indicates that the full distribution of $S_{A \mid B}$ develops long tails with large $S_{A \mid B}$ values when increasing $\Delta J$. For strong disorders $\Delta J \gtrsim 1.5$ we find that $\mathrm{tSDRG}$ is more accurate than vMPS. The vMPS estimates of $S_{A \mid B}$ are consistently below

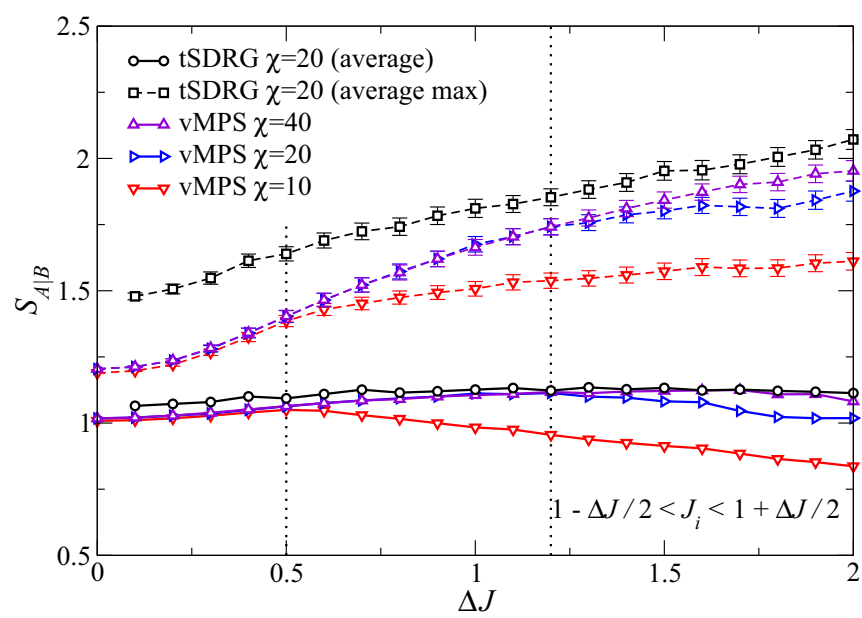

FIG. 11. (Color online) Entanglement entropy $S_{A \mid B}$ for all possible bipartitions (cf. Fig. 6) for $L=30$ as a function of $\Delta J$ averaged over 100 disorder configurations using vMPS and tSDRG. Solid lines indicate the arithmetic mean over disorder configurations while dashed lines denote the mean of the maximal $S_{A \mid B}$ values at the chosen $\Delta J$. Lines connecting symbols are guides to the eye only. Error bars denote standard error of the mean when larger than symbol size. The two vertical dotted lines highlight $\Delta J=0.5$ and 1.2 as discussed in the text. 


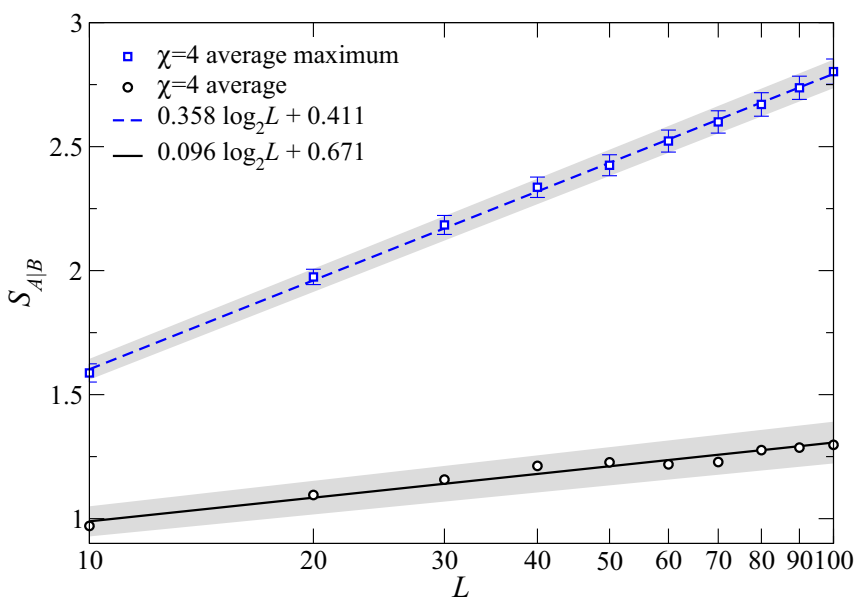

FIG. 12. (Color online) Entanglement entropy $S_{A \mid B}$ as a function of $L$ averaged over 100 samples and all possible bipartitions (as in Fig. 11) for $\chi=4$ and $\Delta J=2^{-}$. The dashed blue line is the fit $(0.358 \pm 0.005) \log _{2} L+(0.41 \pm 0.03)$; the solid black line is $(0.096 \pm 0.008) \log _{2} L+(0.67 \pm 0.04)$. Error bars denote the standard error of the mean for the $S_{A \mid B}$ values when larger than symbol size while gray shaded regions show the standard error of the indicated fits.

the values obtained by the tSDRG. Only when increasing $\chi$ do we reduce the deviation. This behavior is most pronounced for the average of the maximal $S_{A \mid B}$ values. For example, with $\chi=20$, the $S_{A \mid B}$ values obtained for vMPS deviate from the tSDRG results around $\Delta J \approx 1.2$. Hence we see that an increase in $S_{A \mid B}$ requires a considerable increase in $\chi$ for vMPS to accurately capture the entanglement. On the other hand, for weak disorders $\Delta J \lesssim 0.5$, vMPS gives consistent results already for small $\chi=10$. The values obtained for $S_{A \mid B}$ from tSDRG are much higher in this regime. We believe this to be an overestimation of $S_{A \mid B}$ by the tSDRG because, as discussed before, tSDRG selects most strongly the singlet pairs in the disordered system, which of course become less prevalent for low disorder.

Figure 12 shows that when $L$ is increased for $\Delta J=2$, both the average and average peak values of $S_{A \mid B}$ increase logarithmically in $L$. This again implies that as $L$ is increased, the $\chi$ value for vMPS needs to be increased also to be able to capture the entanglement. On the other hand, the holographic nature of the TTN means that the minimal surface in the network increases with system size and thus describes this entanglement without the need to increase $\chi$. Although $S_{A \mid B}$ is therefore captured well by the network, contracting $\rho_{A}$ for larger $L$ becomes increasingly more difficult, even with the simplifications suggested in Sec. IV B, since the size of the matrices scales as $O\left(\chi^{n_{A}}\right)$. We therefore have to restrict ourselves to smaller $\chi$ and $L$ values than in Secs. IV A and IV B.

In Refs. [23,42], Refael and Moore calculate a block entanglement $S_{A, B}$ in the random singlet phase and show that it scales as

$$
S_{A, B} \sim \frac{\log 2}{3} \log _{2} L_{B} \approx 0.231 \ldots \log _{2} L_{B}
$$

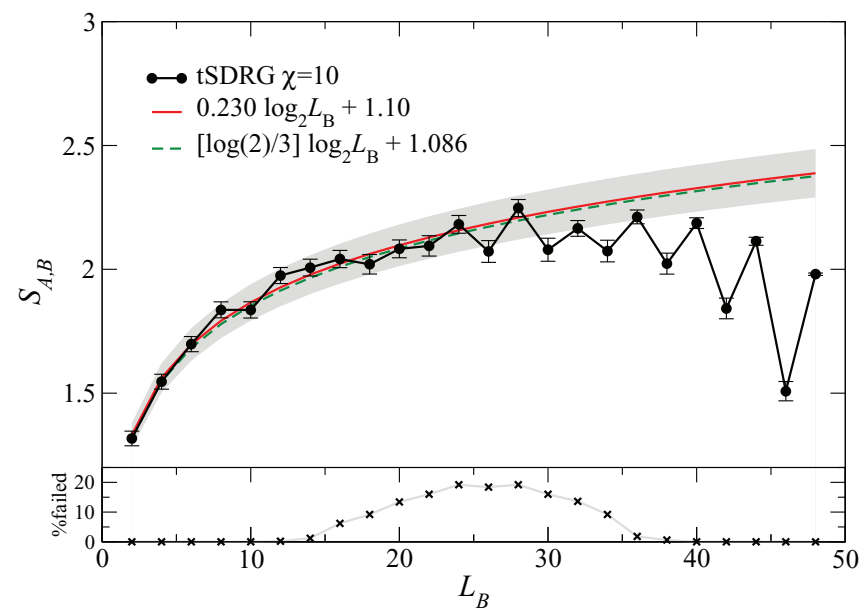

FIG. 13. (Color online) The entanglement entropy $S_{A, B}$ (black) averaged over 500 samples as a function of the size of a block $L_{B}$ placed in the middle of a chain with $L=50$ for $\chi=10$ and $\Delta J=$ $2^{-}$. The fitting (red, solid line) gives $S_{A \mid B}=(0.22 \pm 0.02) \log _{2} L_{B}+$ $(1.12 \pm 0.05)$ for $L_{B} \leqslant 25$, above which finite size effects dominate. The gray-shaded region indicates the accuracy of the fit. The (green) dashed line shows the entanglement scaling (15) from Ref. [42] with the vertical position fitted to the point $L_{B}=2$. The straight black lines are a guide to the eye only. At the bottom, we show the failure rate in percent (crosses) for different $L_{B}$.

where region $B$ is a block of extent $L_{B}$ in the center of the spin chain. Note that this implies an effective central charge [23] of $\tilde{c}=1 \times \log 2$. This is different from the bipartition entanglement $S_{A \mid B}$ that we considered before. We show the resulting $S_{A, B}$ in Fig. 13. The figure clearly indicates that finite size effects become prevalent for large $L_{B}$, so we fit for $L_{B} \leqslant L / 2$ only. The resulting scaling behavior $S_{A, B} \approx$ $(0.22 \pm 0.02) \log _{2} L_{B}$ is consistent with Eq. (15). We note, however, that finite size corrections might still be present at

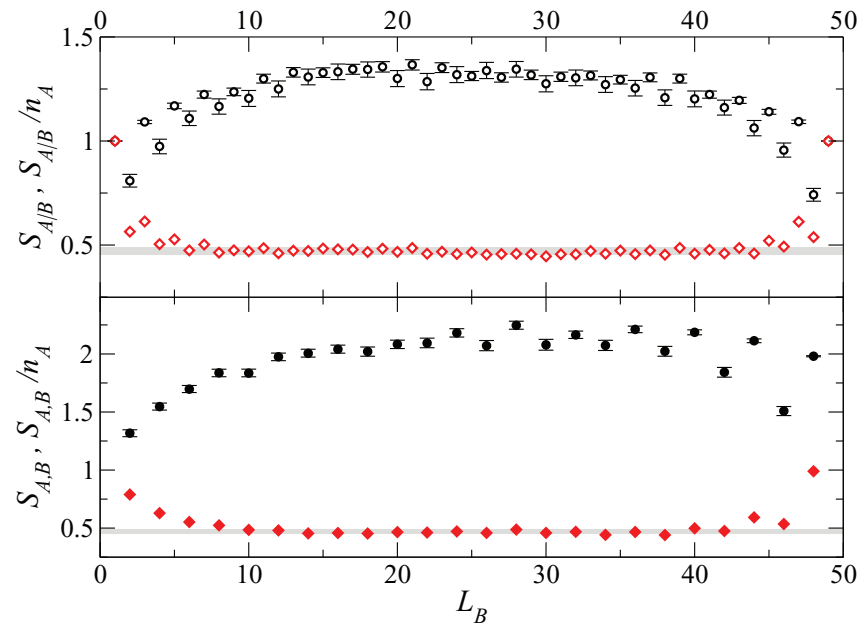

FIG. 14. (Color online) Entanglement entropy $S$ (black circles) and entanglement entropy per bond $S / n_{A}$ (red diamonds) for bipartitions $A \mid B$ (top, open symbols) and blocks $A, B$ (bottom, filled symbols) with $\chi=10$ and $\Delta J=2^{-}$. The entanglement per bond saturates to $0.47 \pm 0.02$ for bipartitions and $0.48 \pm 0.02$ for blocks (gray-shaded regions). 
the system size available to us here; ideally one should aim for much larger system sizes [43].

We finally also examine the entanglement entropy per bond, $S / n_{A}$, of a TTN for both bipartitions $A \mid B$ and blocks $A, B$ with $\chi=10$ when averaging over 500 disorder configurations with $L=50$. Figure 14 shows that away from the boundaries $S / n_{A}$ saturates to the same constant $0.47 \pm 0.02$ for bipartitions and blocks [44]. This is consistent with Ref. [6] and implies that the entanglement entropy is proportional to the length of the holographic minimal surface that connects the two blocks. Note that for $L_{B} \sim L / 2$, we find that up to $20 \%$ of our samples for $\chi=10$ lead to calculations of $S_{A, B}$ consuming memory beyond $100 \mathrm{~GB}$. This is currently out of reach for us and we disregard the configurations. Nevertheless, we think that this is purely a numerical artifact and does not change the average values of $S_{A, B} / n_{A}$ reported here. Calculations with smaller $\chi$ confirm this [44].

\section{CONCLUSION}

In this work, we demonstrate the validity and usefulness of a suitably adaptive tensor network approach to locally disordered one-dimensional quantum many-body systems. In contrast to traditional vMPS approaches to disordered systems, where the initial geometry of the MPS ignores the disorder and only takes it into account at the stage of variational sweeps [45], our approach incorporates the disorder into the fabric of its tensor network. We believe this strategy to be inherently more suited to disordered systems - the results presented here show that the accuracy of tSDRG is already comparable to vMPS without including any additional variational updates. This advantage is particularly evident for long-ranged correlations and an entanglement entropy that violates the area law.

Our results furthermore show that, when disorder averaged, a random AFM spin-1/2 system is well characterized by an effective CFT on the boundary of a discretized holographic bulk. Here we have thus shown the quantitative validity of holography. In particular, our spin-spin correlation function, Fig. 8, as well as the block and bipartition entanglement entropies, Fig. 14, show excellent qualitative and numerical agreement with their holographic counterparts. Such an agreement also reconfirms that the self-assembly of the TTN produces the necessary tensor network geometry.

While here we concentrated on the disordered $X X X$ model, the method should be straightforwardly applicable to the $X X$ and $X X Z$ models as studied by Fisher [21]. Similarly it should work for the Jordan-Wigner transformed equivalent fermionic models with a disordered hopping parameter [46]. It should also be permissible to implement different forms of disorder, such as aperiodic sequences [47] as long as the singlet approximation is valid throughout the renormalization procedure. We have checked that $\mathrm{tSDRG}$, just as the SDRG of Hikihara [25], is also able to model random FM/AFM couplings that create large effective spins as the renormalization progresses. As such it may be possible to use our approach to study higher spin systems given a suitably high $\chi$. It should also be fairly simple to extend the tSDRG method to periodic systems by introducing a bond between the first and last MPO tensor, which is effectively taking a trace over the MPO. We note that implementation of on-site disorder, such as in the random transverse field Ising model [22], does not appear to have a natural implementation using the local RG outlined in Sec. II. Here it may be possible to implement a tensor network with a different structure, but at the moment it is not clear to us how this would be performed.

The tensor network approach makes finding other expectation values, i.e., in addition to those studied here, straightforward as they are simply the contraction of the set of isometries with a matrix operator. An example is the string order parameter [48] that is used to find a hidden topological order in the ground state [49]. If the entanglement entropy can be found, so too can the entanglement spectrum, which has become a popular means of characterizing many-body wave functions [50-56], for better or for worse [57]. Excited states can be found by diagonalizing the top tensor and instead of keeping the lowest energy eigenvector, one keeps a suitable set of higher energy eigenvectors. This will only be accurate for low energy excitations as at each step of the renormalization process only the low energy components are kept, while information about higher energy modes is discarded. Furthermore, it is possible that when moving far away from the ground state the geometry of the network is no longer appropriate.

Our local RG procedure selects spin pairs based on energy gaps. It is tempting to reformulate this based on the local entanglement content of such pairs. However, it is not straightforward to find such a local measure that captures energies and wave functions well simultaneously. In particular, we do not find a convenient local entanglement measure that would have a simple relation to the local values of $J_{i}$. More promising might be the implementation of a variational TTN [30]. Our initial results suggest that this does indeed improve the energy values, but at considerably increased efforts in implementation and computation-every disorder configuration of course necessitating its own variationally updated tree structure.

\section{ACKNOWLEDGMENTS}

We are grateful to Andrew Ferris, Glen Evenbly, José Hoyos, and Nick d'Ambrumenil for valuable discussions. We would like to thank the EPSRC for financial support (EP/J003476/1) and provision of computing resources through the MidPlus Regional HPC Centre (EP/K000128/1). A.M.G. would like to thank the organizers and participants of the Networking Tensor Networks 2012 workshop at the Centro de Ciencias de Benasque.

\section{APPENDIX A: THE NUMERICAL SDRG ALGORITHM}

The algorithm of Ref. [25] can be formulated as follows:

(1) Find the coupling Hamiltonian with the largest gap $\Delta_{i_{m}}$ and create the two-site block.

(2) Diagonalize the two-site block to find the $\chi \leqslant \chi^{\prime}$ lowest eigenvalues $\left(\Lambda_{\chi}\right)$ and corresponding eigenvectors $\left(V_{\chi}\right)$ such that only full $\mathrm{SU}(2)$ multiplets are kept, where $\chi^{\prime}$ is the maximum number of eigenvectors and is set at runtime. 


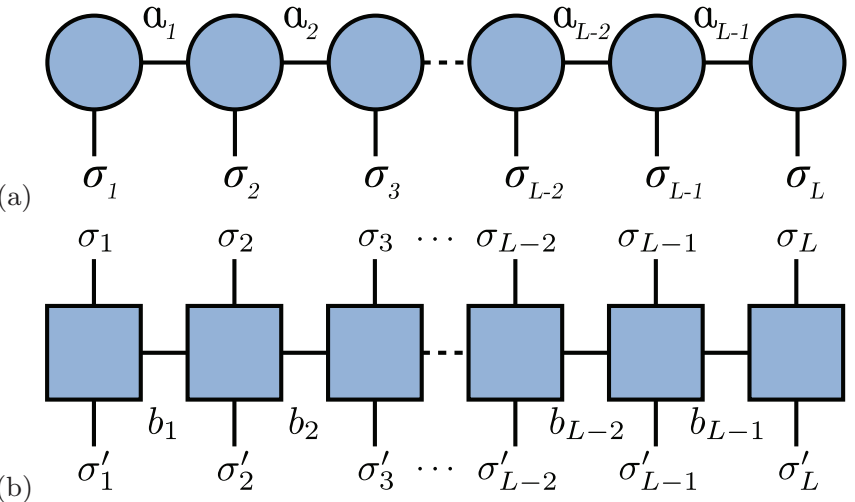

FIG. 15. (Color online) (a) Tensor network diagram of the MPS ket. The circles represent the $M$ tensors in Eq. (B2), the lines are the indices, and connected lines represent tensor contractions. The bra state is the same apart from the vertical lines point upwards and the contents of the tensors are the complex conjugate. (b) Tensor network diagram of the matrix product operator. The $\sigma$ and $\sigma^{\prime}$ legs are physical indices and couple to the tensor network wave function and conjugate. The $b$ are virtual indices and couple the local tensors of the MPO (squares) to each other.

(3) Set the $\chi$ eigenvalues $\left(\Lambda_{\chi}\right)$ from the diagonalization as the new two-site block, which is equivalent to renormalizing the two-site block with $V_{\chi}$,

$$
\tilde{H}_{i_{m}, i_{m}+1}^{B}=V_{\chi}^{\dagger} H_{i_{m}, i_{m}+1}^{B} V_{\chi}=\Lambda_{\chi} .
$$

(4) Renormalize the spin operators on the right- and lefthand sides of the new block to update the couplings

$$
\begin{aligned}
& \overrightarrow{\tilde{s}}_{i_{m}}^{R}=V_{\chi}^{\dagger}\left(\mathbb{1} \otimes \vec{s}_{i_{m}+1}^{R}\right) V_{\chi}, \\
& \overrightarrow{\tilde{s}}_{i_{m}}^{L}=V_{\chi}^{\dagger}\left(\vec{s}_{i_{m}}^{L} \otimes \mathbb{1}\right) V_{\chi} .
\end{aligned}
$$

(5) Diagonalize the neighboring blocks to get the new gaps.

(6) Remove site $i_{m}+1$ and return to step 1 . This process is repeated until the whole system is described by one block.

\section{APPENDIX B: MATRIX PRODUCT OPERATORS AND SDRG}

\section{Matrix product states}

A general wave function describing a spin state on a lattice can be written as

$$
|\Psi\rangle=\sum_{\sigma_{1}, \ldots, \sigma_{L}} C_{\sigma_{1}, \ldots, \sigma_{L}}\left|\sigma_{1}, \ldots, \sigma_{L}\right\rangle,
$$

where $\sigma_{i}$ are the physical indices of the lattice and enumerate the states in the local Hilbert space. The tensor $C_{\sigma_{1}, \ldots, \sigma_{L}}$ can be decomposed into a tensor network, the most common of which is the MPS

$$
|\Psi\rangle=\sum_{\sigma_{1}, \ldots, \sigma_{L}} \sum_{a_{1}, \ldots, a_{L-1}} M_{a_{1}}^{\sigma_{1}} M_{a_{1} a_{2}}^{\sigma_{2}}, \ldots, M_{a_{L-1}}^{\sigma_{L}}\left|\sigma_{1}, \ldots, \sigma_{L}\right\rangle .
$$

Here, $a_{i}=1, \ldots, \chi$ for site $i$ in the bulk. It is convenient when studying tensor networks, such as the MPS, to give the equations a diagrammatic form [Fig. 15(a)]. Each tensor is drawn as a shape where each line coming out represents an index and connected lines represent tensor contractions.

\section{Matrix product operators}

In a similar manner to the matrix product state, operators acting on lattice wave functions can be decomposed into a network of more simple tensors. A general operator on a lattice can be written as

$$
\mathcal{O}=\sum_{\sigma_{1}, \ldots, \sigma_{L}} \sum_{\sigma_{1}^{\prime}, \ldots, \sigma_{L}^{\prime}} D_{\sigma_{1}, \sigma_{1}^{\prime}, \ldots, \sigma_{L}, \sigma_{L}^{\prime}}\left|\sigma_{1}, \ldots, \sigma_{L}\right\rangle\left\langle\sigma_{1}^{\prime}, \ldots, \sigma_{L}^{\prime}\right|
$$

This can be decomposed into a matrix product form to give a MPO:

$$
\begin{aligned}
\mathcal{O}= & \sum_{\sigma_{1}, \ldots, \sigma_{L}} \sum_{\sigma_{1}^{\prime}, \ldots, \sigma_{L}^{\prime}} \sum_{b_{1}, \ldots, b_{L-1}} \\
& \times W_{b_{1}}^{\sigma_{1}, \sigma_{1}^{\prime}} W_{b_{1}, b_{2}}^{\sigma_{2}, \sigma_{2}^{\prime}} \ldots, W_{b_{L-2}, b_{L-1}}^{\sigma_{L-1}, \sigma_{L-1}^{\prime}} W_{b_{L-1}}^{\sigma_{L}, \sigma_{L}^{\prime}} \\
& \times\left|\sigma_{1}, \ldots, \sigma_{L}\right\rangle\left\langle\sigma_{1}^{\prime}, \ldots, \sigma_{L}^{\prime}\right|
\end{aligned}
$$

where there are two sets of physical indices $\sigma$ and $\sigma^{\prime}$, which connect to the bra and ket states, respectively. Figure 15(b) gives the pictorial form of the MPO.

The Heisenberg Hamiltonian on an open lattice,

$$
H_{X X X}=\sum_{i=1}^{L-1} J_{i}\left[\frac{1}{2}\left(s_{i}^{+} s_{i+1}^{-}+s_{i}^{-} s_{i+1-1}^{+}\right)+s_{i}^{z} s_{i+1}^{z}\right]
$$

can be encoded as an MPO with

$$
\begin{gathered}
W_{b_{1}}=\left(\begin{array}{ccccc}
\mathbb{1} & \frac{J_{1}}{2} s_{1}^{+} & \frac{J_{1}}{2} s_{1}^{-} & J_{1} s_{1}^{z} & 0
\end{array}\right), \\
W_{b_{i-1}, b_{i}}=\left(\begin{array}{ccccc}
\mathbb{1} & \frac{J_{i}}{2} s_{i}^{+} & \frac{J_{i}}{2} s_{i}^{-} & J_{i} s_{i}^{z} & 0 \\
0 & 0 & 0 & 0 & s_{i}^{-} \\
0 & 0 & 0 & 0 & s_{i}^{+} \\
0 & 0 & 0 & 0 & s_{i}^{z} \\
0 & 0 & 0 & 0 & \mathbb{1}
\end{array}\right), \\
W_{b_{L-1}}=\left(\begin{array}{c}
0 \\
s_{L}^{-} \\
s_{L}^{+} \\
s_{L}^{z} \\
\mathbb{1}
\end{array}\right) .
\end{gathered}
$$

Simply multiplying $W_{b_{1}} W_{b_{1}, b_{2}} \cdots W_{b_{L-2}, b_{L-1}} W_{b_{L-1}}$ results in (B5). The top right element of Eq. (B7) and equivalent elements in Eqs. (B6) and (B8) are referred to as the on-site elements. This is where an external magnetic field of the form $h_{i} S_{i}^{z}$ would be introduced. Furthermore, it is possible to include longer range interactions in the elements away from the top and right row and column [58,59].

Another way of describing the contents of an MPO is a matrix product (MP) diagram [58]. This is a pictorial representation of the elements in the tensor, whereby the indices are numbered circles and the corresponding elements are paths that connect any two indices (Fig. 16). Matrix multiplication, or contraction, is then represented by the sum 

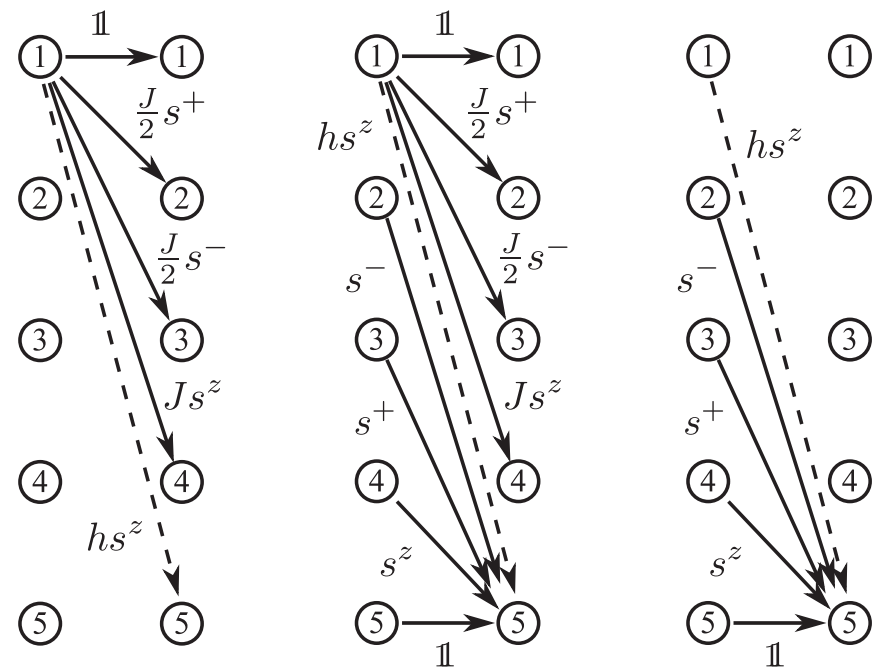

FIG. 16. The left, center, and right are MP diagrammatic forms of Eqs. (B6), (B7), and (B8), respectively. The circles represent the virtual indices $\left(b_{i-1}, b_{i}\right)$ of the MPO tensor and arrows show the corresponding operator. The dashed arrows highlight the possibility of an additional magnetic field operator $h S^{z}$ not present in (B6), (B7), and (B8).

of the unique paths that connect the indices on the far left and right of the diagram when multiple matrices are placed end

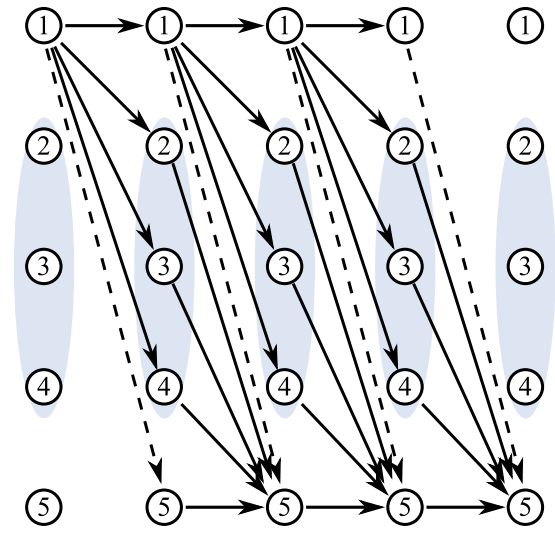

FIG. 17. (Color online) MP diagram of the contraction of a foursite MPO of the Heisenberg Hamiltonian (B5). Symbols and lines as in Fig. 16. The shaded ellipses linking tensor entries 2, 3, and 4 corresponds to the simplifications employed in Fig. 3.

to end (Fig. 17). For MPOs it is understood that the binary operator between terms is a tensor product. Tracing out the different paths in Fig. 17 results in the standard form (B5) with $L=4$. The MP diagrams give a convenient means of visualizing the components of the MPO and are particularly useful when creating operators with long-range components or periodic boundary conditions.
[1] J. McGreevy, Adv. High Energy Phys. 2010, 723105 (2010).

[2] J. Maldacena, Int. J. Theor. Phys. 38, 1113 (1999).

[3] B. Swingle, Phys. Rev. D 86, 065007 (2012).

[4] J. Molina-Vilaplana, J. High Energy Phys. 05 (2013) 024.

[5] S. Ryu and T. Takayanagi, Phys. Rev. Lett. 96, 181602 (2006).

[6] G. Evenbly and G. Vidal, J. Stat. Phys. 145, 891 (2011).

[7] S. R. White, Phys. Rev. Lett. 69, 2863 (1992).

[8] S. Östlund and S. Rommer, Phys. Rev. Lett. 75, 3537 (1995).

[9] U. Schollwöck, Ann. Phys. (NY) 326, 96 (2011).

[10] S. Sharma and G. K.-L. Chan, J. Chem. Phys. 136, 124121 (2012).

[11] D. Perez-Garcia, F. Verstraete, M. M. Wolf, and J. I. Cirac, Quantum Inf. Comput. 7, 401 (2007).

[12] M. Cramer, A. Flesch, I. P. McCulloch, U. Schollwöck, and J. Eisert, Phys. Rev. Lett. 101, 063001 (2008).

[13] G. Vidal, Phys. Rev. Lett. 99, 220405 (2007).

[14] F. Verstraete and J. I. Cirac, arXiv:cond-mat/0407066.

[15] G. Evenbly and G. Vidal, Phys. Rev. B 81, 235102 (2010).

[16] Z. Y. Xie, J. Chen, M. P. Qin, J. W. Zhu, L. P. Yang, and T. Xiang, Phys. Rev. B 86, 045139 (2012).

[17] S. Dusuel, M. Kamfor, R. Orús, K. P. Schmidt, and J. Vidal, Phys. Rev. Lett. 106, 107203 (2011).

[18] F. Iglói and C. Monthus, Phys. Rep. 412, 277 (2005).

[19] S. K. Ma, C. Dasgupta, and C. K. Hu, Phys. Rev. Lett. 43, 1434 (1979).

[20] C. Dasgupta and S. K. Ma, Phys. Rev. B 22, 1305 (1980).

[21] D. S. Fisher, Phys. Rev. B 50, 3799 (1994).

[22] D. S. Fisher, Phys. Rev. B 51, 6411 (1995).

[23] G. Refael and J. E. Moore, Phys. Rev. Lett. 93, 260602 (2004).
[24] E. Westerberg, A. Furusaki, M. Sigrist, and P. A. Lee, Phys. Rev. Lett. 75, 4302 (1995).

[25] T. Hikihara, A. Furusaki, and M. Sigrist, Phys. Rev. B 60, 12116 (1999).

[26] E. Westerberg, A. Furusaki, M. Sigrist, and P. A. Lee, Phys. Rev. B 55, 12578 (1997).

[27] K. G. Wilson, Rev. Mod. Phys 47, 773 (1975).

[28] G. Evenbly and G. Vidal, Phys. Rev. B 79, 144108 (2009).

[29] Y. Y. Shi, L. M. Duan, and G. Vidal, Phys. Rev. A 74, 022320 (2006).

[30] L. Tagliacozzo, G. Evenbly, and G. Vidal, Phys. Rev. B 80, 235127 (2009).

[31] J. I. Cirac and F. Verstraete, J. Phys. A: Math. Gen. 42, 504004 (2009).

[32] F. Verstraete, V. Murg, and J. I. Cirac, Adv. Phys. 57, 143 (2008).

[33] G. Vidal, arXiv:0707.1454v2.

[34] Let us emphasize that we expect a variational tSDRG to be at least as good as our vMPS. Here, however, we concentrate predominately on showing the validity and usefulness of a TTN approach to disordered chains. In order to retain clarity, we thus refrain from increasing algorithmic complexity.

[35] J. Voit, Rep. Prog. Phys. 58, 977 (1995).

[36] The asymptotic behavior for the clean Heisenberg $X X X$ model [60] is $\left\langle s_{r} s_{0}\right\rangle \sim(-1)^{r} \sqrt{\log r} / r$. The difference in power of the algebraic decay points to the very different origin of Eq. (12).

[37] The fitting was performed using the lsqcurvefit function in MATLAB version 2013a. The function is based on a trust-region algorithm [61] with weights to take into account the accuracy of the data. 
[38] J. A. Hoyos, A. P. Vieira, N. Laflorencie, and E. Miranda, Phys. Rev. B 76, 174425 (2007).

[39] N. Laflorencie, Phys. Rev. B 72, 140408 (2005).

[40] L. Bombelli, R. K. Koul, J. Lee, and R. D. Sorkin, Phys. Rev. D 34, 373 (1986).

[41] J. Eisert, M. Cramer, and M. B. Plenio, Rev. Mod. Phys. 82, 277 (2010).

[42] G. Refael and J. E. Moore, Phys. Rev. B 76, 024419 (2007).

[43] F. Iglói and Y.-C. Lin, J. Stat. Mech.: Theor. Exp. (2008) P06004.

[44] We find $0.42 \pm 0.02$ for $\chi=4$ for both blocks and bipartitions. This might conceivably suggest that $S / n_{A}=0.5$ as limiting value for larger $\chi$ and $L$. In turn, this would imply $n_{A}=$ $2 \log L_{B} / 3$. The failure rate for these calculations is $<1 \%$.

[45] K. A. Hallberg, Adv. Phys. 55, 477 (2006).

[46] G. Ramírez, J. Rodríguez-Laguna, and G. Sierra, arXiv:1402.5015.

[47] R. Juhász and Z. Zimborás, J. Stat. Mech.: Theor. Exp. (2007) P04004.

[48] M. den Nijs and K. Rommelse, Phys. Rev. B 40, 4709 (1989).

[49] P. Lajkó, E. Carlon, H. Rieger, and F. Iglói, Phys. Rev. B 72, 094205 (2005).
[50] H. Li and F. D. M. Haldane, Phys. Rev. Lett. 101, 010504 (2008).

[51] N. Regnault, B. A. Bernevig, and F. D. M. Haldane, Phys. Rev. Lett. 103, 016801 (2009).

[52] F. Pollmann, A. M. Turner, E. Berg, and M. Oshikawa, Phys. Rev. B 81, 064439 (2010).

[53] A. M. Läuchli, E. J. Bergholtz, J. Suorsa, and M. Haque, Phys. Rev. Lett. 104, 156404 (2010).

[54] V. Alba, M. Haque, and A. M. Läuchli, J. Stat. Mech. Theor. Exp. (2012) P08011.

[55] X. Deng, R. Citro, E. Orignac, A. Minguzzi, and L. Santos, New J. Phys. 15, 045023 (2013).

[56] L. Lepori, G. De Chiara, and A. Sanpera, Phys. Rev. B 87, 235107 (2013).

[57] A. Chandran, V. Khemani, and S. L. Sondhi, arXiv:1311.2946.

[58] G. M. Crosswhite and D. Bacon, Phys. Rev. A 78, 012356 (2008).

[59] F. Fröwis, V. Nebendahl, and W. Dür, Phys. Rev. A 81, 062337 (2010).

[60] I. Affleck, J. Phys. A: Math. Gen. 31, 4573 (1998).

[61] J. Moré and D. Sorensen, SIAM J. Sci. Stat. Comput. 4, 553 (1983). 